\title{
PRODUTIVIDADE DE MINICEPAS DE CEDRO AUSTRALIANO E REMOÇÃO DE NUTRIENTES PELA COLETA SUCESSIVA DE MINIESTACAS
}

\section{PRODUCTIVITY OF AUSTRALIAN CEDAR MINISTUMPS AND NUTRIENTS REMOVAL BY SUCCESSIVE COLLECTION OF MINICUTTINGS}

\author{
Juliana Sobreira de Souza ${ }^{1}$ Deborah Guerra Barroso ${ }^{2}$ Mírian Peixoto Soares da Silva ${ }^{3}$ \\ Daniele de Alvarenga Ferreira ${ }^{4}$ Geraldo de Amaral Gravina ${ }^{5}$ José Geraldo de Araújo Carneiro ${ }^{6}$
}

\section{RESUMO}

O manejo de minicepas para produção de miniestacas resulta na remoção contínua de nutrientes, sendo indispensável sua reposição para evitar o esgotamento e queda na produção. Assim, é necessário determinar a quantidade de nutrientes removidos no tempo, em diferentes modelos de manejo, para viabilizar a produção comercial. O objetivo deste trabalho foi avaliar dois sistemas de minijardim multiclonal de Toona ciliata, conduzidos em tubete e canaletão, quanto à produtividade e remoção de nutrientes ao longo do tempo pelas minicepas, produzidas por via seminal, submetidas a coletas sucessivas de miniestacas. Foram utilizadas 186 mudas em cada sistema de manejo. Foram determinados os conteúdos de nutrientes nas brotações emitidas por 20 minicepas selecionadas ao acaso em cada sistema. Foram realizadas cinco coletas de brotações em intervalos médios de 32 dias no sistema canaletão, enquanto no sistema tubete foram realizadas três coletas de brotações em intervalos médios de 31 dias. Os dados foram analisados por amostragem simples ao acaso considerando uma população infinita de miniestacas. As médias dos tratamentos qualitativos foram comparadas por intervalo de confiança, utilizando o teste " $t$ " de Student, a $5 \%$ de probabilidade, e os tratamentos quantitativos (produtividade e nutrientes removidos no tempo pelas minicepas) por regressão. Foi constatado que a produtividade e o conteúdo de nutrientes removidos pelas minicepas manejadas em canaletão foram superiores à dos tubetes. O nitrogênio e o potássio foram os nutrientes mais removidos pelas minicepas em ambos os sistemas de manejo. São apresentadas as equações que descrevem as quantidades removidas ao longo do tempo nos dois sistemas.

Palavras-chave: Toona ciliata; minijardim multiclonal; produção de mudas.

\section{ABSTRACT}

The management of ministumps to produce minicuttings results in the continuous removal of nutrients and it is necessary replacement to avoid depletion and decline in production. Thus, it is necessary to determine the amount of nutrients removed over time, in different management models, to enable commercial

1 Licenciada em Biologia, Msc., Doutoranda do Programa de Pós-Graduação em Ecologia e Recursos Naturais, Universidade Estadual do Norte Fluminense Darcy Ribeiro, Centro de Biociências e Biotecnologia, Laboratório de Ciências Ambientais (LCA), Av. Alberto Lamego, 2000, Parque Califórnia, CEP 28013-602, Campos dos Goytacazes (RJ), Brasil. julianauenf@gmail.com

2 Engenheira Agrônoma, $\mathrm{Dr}^{\mathrm{a}}$, Professora de Silvicultura, Universidade Estadual do Norte Fluminense Darcy Ribeiro, Centro de Ciências e Tecnologias Agropecuárias, Laboratório de Fitotecnia (LFIT), Av. Alberto Lamego, 2000, Parque Califórnia, CEP 28013-602, Campos dos Goytacazes (RJ), Brasil. deborah@uenf.br

3 Engenheira Agrônoma, Msc., Doutoranda do Programa de Pós-Graduação em Produção Vegetal, Universidade Estadual do Norte Fluminense Darcy Ribeiro, Centro de Ciências e Tecnologias Agropecuárias, Laboratório de Fitotecnia (LFIT), Av. Alberto Lamego, 2000, Parque Califórnia, CEP 28013-602, Campos dos Goytacazes (RJ), Brasil.mirianpsoares@gmail.com

4 Engenheira Agrônoma, Msc., Viveirista, CEP 28360-000, Bom Jesus do Itabapoana (RJ), Brasil. ferreira.da@gmail.com

5 Engenheiro Agrônomo, Licenciado em Ciências Agrícolas, Dro, Professor Associado, Universidade Estadual do Norte Fluminense Darcy Ribeiro, Centro de Ciências e Tecnologias Agropecuárias, Laboratório de Engenharia Agrícola (LEAG), Av. Alberto Lamego, 2000, Parque Califórnia, CEP 28013-602, Campos dos Goytacazes (RJ), Brasil.gravina@uenf.br

6 Engenheiro Florestal, PhD., Pesquisador Associado, Universidade Estadual do Norte Fluminense Darcy Ribeiro, Centro de Ciências e Tecnologias Agropecuárias, Laboratório de Fitotecnia (LFIT), Av. Alberto Lamego, 2000, Parque Califórnia, CEP 28013-602, Campos dos Goytacazes (RJ), Brasil. carneiro@uenf.br

Recebido para publicação em 20/04/2010 e aceito em 31/08/2012 
production. The objective of this study was to evaluate two minigarden multiclonal systems of Toona ciliata, in plastic tubes and bed, for productivity and nutrients removal over time by ministumps, produced by seeds, submitted to successive collections of minicuttings. One hundred and eighty-six seedlings were used in each management system. In this paper, it was determined the nutrient content in shoots issued by 20 ministumps randomly selected in each system. Five shoots were collected at intervals of 32 days in bed system, while in the plastic tubes system three shoots were collected at intervals of 31 days. The data were analyzed by a sample simple random considering an infinite population of minicuttings. The qualitative treatment average was compared by confidence interval using the Student $t$ test, 5\% probability, and the quantitative treatment (productivity and nutrients removed in time by ministumps) by regression. It has been found that the productivity and nutrient contents removed by ministumps in bed were higher than in the plastic tubes. Nitrogen and potassium are the nutrients most removed by ministumps in both systems. Here, it was presented the curves corresponding to the nutrients exported, to estimate the replacement that will be made in each system over time.

Keywords: Toona ciliata; multiclonal minigarden; seedling production.

\section{INTRODUÇÃO}

A Toona ciliata (MURAKAMI, 2008) é uma espécie arbórea exótica no Brasil, que vem, a cada dia, chamando a atenção de produtores e ganhando o seu espaço no mercado brasileiro, por ser uma alternativa lucrativa e apresentar crescimento rápido e madeira semelhante à do cedro brasileiro (Cedrella fissilis) (PINHEIRO et al., 2003; PAIVA et al., 2007), indicada para a fabricação de móveis finos e acabamentos em construção civil (MURAKAMI, 2008).

No Brasil, essa espécie encontrou condições favoráveis para sua adaptação, aliada a resistência à Hypsipyla grandella (LIAO et al., 2007; MURAKAMI, 2008), uma broca que ataca o broto terminal do cedro-rosa (LIAO et al., 2007; MURAKAMI, 2008). O método de propagação comercial de Toona ciliata é via seminal, entretanto, a oferta sazonal das sementes e sua curta viabilidade ao longo do tempo, representam um problema para a oferta contínua de mudas para implantação de povoamentos (SOUZA et al., 2009). A propagação vegetativa da Toona ciliata vem sendo testada com sucesso através da miniestaquia (SOUZA et al., 2009) e da propagação in vitro (MROGINSKI et al., 2003).

Para a implantação de minijardins clonais, vários sistemas de produção podem ser utilizados, entre os quais se destacam a condução em canaletões, que são canteiros suspensos, sem restrição do sistema radicular das minicepas, e em tubetes, que permitem o manejo individualizado das minicepas (HIGASHI et al., 2000; HIGASHI et al., 2002).

O fornecimento dos nutrientes nos minijardins pode ser feito por adubação dos substratos ou por hidroponia nos canaletões preenchidos com areia como substrato, sendo os nutrientes necessários ao crescimento das brotações fornecidos diariamente com a irrigação (WENDLING et al., 2005).

Embora a miniestaquia tenha se mostrado viável para a propagação de Toona ciliata, em função da sua alta capacidade de rebrota (SOUZA et al., 2009), pouco se sabe sobre a extração de nutrientes pelas minicepas ao longo de coletas sucessivas, cuja reposição poderá favorecer a produtividade dos sistemas, bem como a qualidade das mudas a serem produzidas.

O objetivo deste trabalho foi comparar a produtividade de minicepas de Toona ciliata conduzidas em canaletão e tubetes e determinar a remoção de nutrientes durante o manejo do minijardim nos dois sistemas.

\section{MATERIAL E MÉTODOS}

O experimento foi conduzido na Unidade de Apoio à Pesquisa (UAP) da Universidade Estadual do Norte Fluminense (UENF), Campos dos Goytacazes/ RJ. O experimento teve início em fevereiro de 2009 e o encerramento em novembro de 2009.

A semeadura foi efetuada de Toona ciliata em 1000 tubetes plásticos, com $180 \mathrm{~cm}^{3}$ de volume, contendo mistura composta por substrato comercial (Plantmax ${ }^{\circledR}$ florestal), fibra de coco e torta de filtro de cana-de-açúcar, na proporção de 2:1:1 v/v, respectivamente, sendo colocadas três sementes por recipiente. Foram adicionados ao substrato $2,2 \mathrm{Kg} \mathrm{m}^{-3}$ de ureia revestida, conforme a dose aplicada para citros (AZEVEDO et al., 2009) e 1,5 $\mathrm{Kg} \mathrm{m}^{-3}$ de superfosfato simples. A semeadura foi realizada em 11 de fevereiro de 2009.

Foram selecionadas 372 mudas produzidas nos tubetes para a implantação dos minijardins mul- 
ticlonais (canaletão e tubete), sendo a metade repicada para o canaletão dois meses após a semeadura.

Foram realizadas cinco coletas de brotações em intervalo médio de 32 dias no sistema canaletão, enquanto no sistema tubete foram realizadas três coletas de brotações em intervalo médio de 31 dias.

O minijardim multiclonal em tubetes e em canaletão localizaram-se em condições de estufa. Cada sistema de manejo foi constituído de 186 minicepas. Aos 96 dias após a semeadura (DAS), essas 186 mudas de ambos os sistemas de produção, foram recepadas a altura de $8 \mathrm{~cm}$ a partir do colo das mudas, para obtenção das minicepas, responsáveis pela emissão das brotações.

Foi coletada a parte apical (acima de $8 \mathrm{~cm}$ de altura das mudas) de 20 mudas de cada sistema de manejo para análise nutricional. Após a primeira recepagem, as minicepas forneceram brotações, sendo coletadas 20 amostras de cada sistema de manejo para avaliação nutricional, identificando-se as quantidades dos nutrientes removidos. O número e intervalo entre coletas foram determinados em função do crescimento das brotações. Foram realizadas seis coletas (incluindo a coleta da parte apical no momento da poda apical) no sistema canaletão com a seguinte periodicidade: 96, 146, 182, 211, 245, 273 DAS $(0,50,86,115,149$ e 177 DAR), enquanto no sistema de tubetes (incluindo a coleta da parte apical no momento da poda apical) foram realizadas quatro coletas com a seguinte periodicidade: 96, 211, 245, 273 DAS (0, 115, 149 e 177 DAR). Após a primeira coleta de brotações, as minicepas dos tubetes só emitiram brotações mediante a aplicação semanal de sulfato de amônio. A seleção das 20 minicepas de cada sistema de manejo foi realizada aleatoriamente na primeira coleta apical das mudas. Após a coleta, essas minicepas foram identificadas com etiquetas, de forma que a cada coleta, as 20 brotações colhidas de cada sistema de manejo para análise fossem provenientes das mesmas minicepas durante todo o experimento.

Foi aplicado nas minicepas manejadas em tubetes $6,5 \mathrm{~g} \mathrm{~L}^{-1}$ de $\left(\mathrm{NH}_{4}\right)_{2} \mathrm{SO}_{4}$ semanalmente a partir dos 79 dias DAR.

Após cada coleta, a parte apical das mudas e as brotações foram acondicionadas em sacos de papel, e colocadas para secar em estufa de circulação forçada de ar, a $70^{\circ} \mathrm{C}$, durante $72 \mathrm{~h}$. Após secagem, a parte apical das mudas e as brotações foram pesadas para determinação da massa seca e trituradas em moinho tipo Wiley, usando peneira de
20 mesh e, depois, foram acondicionadas em recipientes plásticos hermeticamente fechados.

Posteriormente, o material triturado foi submetido a análises químicas para determinação dos teores de nitrogênio $\left(\mathrm{N}_{\text {org }}\right)$, fósforo $(\mathrm{P})$, potássio $(\mathrm{K})$, cálcio $(\mathrm{Ca})$, magnésio $(\mathrm{Mg})$ e enxofre (S), no setor de Nutrição Mineral de Plantas do Laboratório de Fitotecnia/CCTA/UENF. As análises foram realizadas de acordo com metodologias descritas por Malavolta et al. (1997). Após digestão sulfúrica $\left(\mathrm{H}_{2} \mathrm{SO}_{4}\right.$ e $\left.\mathrm{H}_{2} \mathrm{O}_{2}\right)$ o $\mathrm{N}$ foi determinado pelo método de Nessler, o P pela determinação colorimétrica, pelo método azul de molibdato, e o K por espectrofotometria de emissão de chama.

Os nutrientes $\mathrm{Ca}, \mathrm{Mg}, \mathrm{S}$ foram quantificados em extratos obtidos após oxidação do material vegetal pela digestão nitroperclórica $\left(\mathrm{HNO}_{3}\right.$ e $\left.\mathrm{HClO}_{4}\right)$. O Ca e o $\mathrm{Mg}$ foram determinados por espectrofotometria de absorção atômica no modelo Zeiss AAS4. O S foi determinado por turbidimetria com cloreto de bário.

Para os dados acumulados de massa seca e nutrientes removidos no tempo pelas minicepas, foi considerado o delineamento experimental inteiramente casualizado com 20 repetições, sendo cada planta uma repetição. As variáveis: massa seca, $\mathrm{N}$, $\mathrm{P}, \mathrm{K}, \mathrm{Ca}, \mathrm{Mg}$, e $\mathrm{S}$ foram avaliadas durante 6 épocas no sistema de canaletão e em 4 épocas no sistema em tubetes, totalizando um período de 273 dias em cada sistema. Depois de verificada a significância do fator épocas de colheita na análise de variância, os graus de liberdade de épocas foram desdobrados e analisados por modelos de regressão linear, sendo que a escolha do modelo de regressão de melhor ajuste aos dados baseou-se na significância da análise de variância da regressão; no coeficiente de determinação $\left(\mathrm{R}^{2}\right)$; no teste de significância dos coeficientes da regressão (teste " $t$ " de Student); e no diagrama de dispersão dos dados e dos resíduos. Para verificar a adequação das equações de regressão adotadas nos sistemas de canaletão e de tubete foram testadas as igualdades de seus coeficientes, também pelo teste "t" de Student. Em todos os testes foram utilizados os níveis de 5 e de $1 \%$ de significância.

\section{RESULTADOS E DISCUSSÃO}

Com relação à produção de miniestacas por minicepa no sistema de tubete, a Figura 1 permite visualizar uma tendência cíclica entre as coletas, em que a produção inicial foi baixa (211 dias após 
a semeadura - DAS), aumentando na segunda coleta, com 1,35 miniestacas por minicepa e caindo novamente na terceira coleta, cujo intervalo foi seis dias menor. O comportamento cíclico na produção de miniestacas pode estar relacionado à exaustão temporária das minicepas, ocasionando menores produções (TITON et al., 2003). Este efeito cíclico também foi observado por Xavier et al. (2003) e Rosa et al. (2009), com Cedrella fissilis, e Eucalyptus dunnii, respectivamente.

No sistema em canaletão também foi possível observar a grande heterogeneidade entre as médias de produção de miniestacas por minicepa de Toona ciliata (Figura 1), que variaram de 1,7 a 3,3. A $4^{\text {a }}$ coleta (245 DAS) de miniestacas apresentou resultados superiores de produção, não diferindo da coleta seguinte. Cunha et al. (2005) constataram um aumento na produção de miniestacas na coleta três e quatro de Eucalyptus benthamii. Titon et al. (2003) realizaram oito coletas de miniestacas de Eucalyptus grandis e observaram nas coletas de número $4 \mathrm{e}$ 5 ligeiro decréscimo nos valores de produção, sendo tais valores, a partir da sexta coleta, maiores e mais estáveis até a realização da última coleta. Esse comportamento pode ser resultado de uma adaptação das minicepas à quebra de dominância apical após a primeira poda e a reorganização do sistema de crescimento ortotrópico para um sistema plagiotrópico, desse modo, após a primeira coleta, as gemas dormentes tornaram-se ativas, resultando em maior

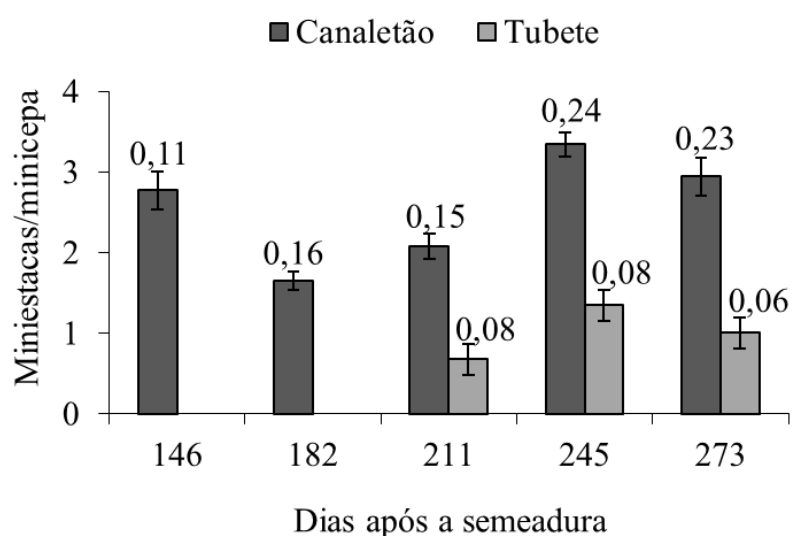

FIGURA 1: Produtividade de minicepas de Toona ciliata conduzidas em dois sistemas de minijardim multiclonal (Barras representam o $\mathrm{IC}_{95 \%}$ )

FIGURE 1: Productivity of ministumps of Toona ciliata conducted in two minigarden multiclonal systems. (Bars represent $\mathrm{CI}_{95 \%}$ ) estímulo ao crescimento (WENDLING e SOUZA JÚNIOR, 2003).

A média geral de produção de miniestacas por minicepa igual a 1,0 no sistema de tubete (Figura 1), no intervalo médio de 31 dias em três coletas, encontra-se abaixo dos resultados observados por Titon et al. (2003), para Eucalyptus grandis em intervalos médios de 7 dias em oito coletas; por Cunha et al. (2005), para Eucalyptus benthamii em intervalos de 25 a 30 dias em cinco coletas, por Rosa (2006), para Eucalyptus dunnii em intervalos de 23 dias em 14 coletas e Souza Junior et al. (2008), para Grevillea robusta em intervalos de 25 dias em 15 coletas, que obtiveram a produção de 7,5; 4,1; 2,8 e 1,7 miniestacas por minicepa, respectivamente, neste sistema de minijardim. Porém, este valor médio de produção é semelhante aos resultados observados por Xavier et al. (2003) e Cunha et al. (2008), com produção média de 1,3 miniestacas por minicepa para Cedrella fissilis e Erythrina falcata, em intervalos regulares de 30 dias em quatro coletas para ambas as espécies. Diferenças podem ser atribuídas a características da espécie, juntamente a condições climáticas e manejo.

A média geral de 2,6 miniestacas de Toona ciliata por minicepa (Figura 1) em intervalos médios de 32 dias entre as cinco coletas no sistema de canaletão foi inferior à encontrada por Cunha et al. (2008), Wendling et al. (2007) e por Cunha et al. (2005), que obtiveram produção de 2,9; 4,4 e 8,1 para Erythrina falcata, Ilex paraguariensis e Eucalyptus benthamii a cada de 15 dias em oito coletas, 39,1 dias em 11 coletas e 25 a 30 dias em cinco coletas, respectivamente.

A produtividade média das minicepas produzidas nos canaletões foi superior ao sistema tubete (Figura 1), indicando um possível aumento no que tange à produção. Resultados semelhantes foram obtidos por Cunha et al. (2005) e Cunha et al. (2008) ao trabalharem com Eucalyptus benthamii e Erythrina falcata, respectivamente. Os autores observaram que o sistema em canaletão produziu maior quantidade de miniestacas, em comparação ao sistema em tubetes.

Cunha et al. (2005), trabalhando com Eucalyptus benthamii, ao compararem esses dois sistemas também obtiveram resultados superiores para o sistema de minijardim clonal em canaletão. Esta diferença associa-se ao aumento da área para absorção de nutrientes fornecido às minicepas neste sistema. Esses autores sugerem que intervalos de 25 a 30 dias em sistemas de tubete e canaletão podem vir a ser interessantes, uma vez que o número 
de intervenções com as minicepas é reduzido, com isso diminui o estresse e o consumo de mão de obra na produção de mudas é menor. Vale salientar que para algumas espécies não é possível diminuir este intervalo, pois as mesmas precisam de mais tempo para formar novas brotações aptas a serem usadas na miniestaquia, como observado para as minicepas de Toona ciliata.

Resultados semelhantes foram obtidos por Cunha et al. (2008) ao trabalharem com Erythrina falcata. Os autores observaram que o sistema em canaletão produziu maior quantidade de miniestacas, em comparação ao sistema em tubetes.

Para os dois sistemas de produção, nota-se uma tendência alternada na produção de miniestacas (Figura 1), atingindo o ponto máximo na $2^{\mathrm{a}}$ coleta de miniestacas $(1,4)$ para tubete, enquanto para o canaletão valores superiores na $4^{\text {a }}$ coleta de brotações $(3,3)$. Esta diferença entre uma coleta e outra, em relação à produção, pode ocorrer em sistemas de minijardins (TITON et al., 2003). A reposição das perdas nutricionais poderia contribuir para uma produção mais constante ao longo do tempo.

Na Figura 2 são apresentados os resultados referentes ao ajuste de regressão para a produção acumulada das miniestacas $/ \mathrm{m}^{2}$ de Toona ciliata em função dos DAS. Ao longo das coletas, a produção de miniestacas $/ \mathrm{m}^{2}$ no sistema canaletão foi superior ao sistema de tubetes, mostrando que, mesmo com densidade inferior de minicepas por $\mathrm{m}^{2}$, o sistema de canaletão é mais produtivo.

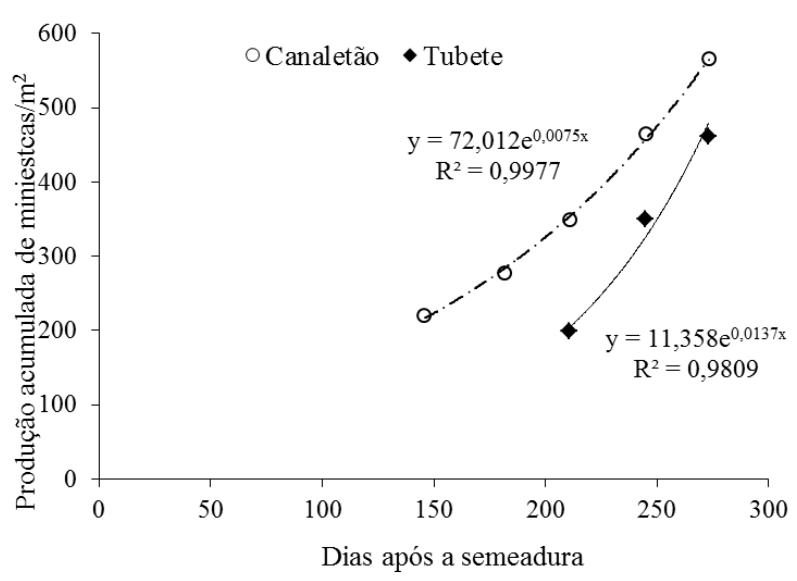

FIGURA 2: Produção acumulada de miniestacas de Toona ciliata conduzidas em dois sistemas de minijardim multiclonal.

FIGURE 2: Cumulative production of Toona ciliata minicuttings conducted in two multiclonal minigarden.
Houve comportamento exponencial na produtividade acumulada dos sistemas (Figura 2). A figura abaixo (Figura 2) mostra que não há exaustão das minicepas com o decorrer do tempo, desde que o fornecimento de nutrientes seja mantido nos tubetes. Ferreira (2009), trabalhando com a mesma espécie, utilizando outro tipo de substrato, ao observar sintomas visuais de deficiência nutricional, iniciou a aplicação de solução nutritiva nas minicepas manejadas em canaletão aos 62 dias após a recepagem.

Conforme as Figuras 3 e 4, as equações de regressão para o sistema em tubetes diferiram das equações do sistema em canaletão, pela comparação de suas taxas de inclinação (coeficiente) para massa seca, $\mathrm{N}, \mathrm{P}, \mathrm{Ca}, \mathrm{Mg}$, e $\mathrm{S}$, pelo teste " $\mathrm{t}$ " para diferença dos "betas". Para o K, os modelos escolhidos já foram diferentes para cada sistema.

Como se pode observar na Figura 3, o acúmulo de massa seca por minicepas ao longo do manejo foi maior no sistema de canaletão.

O sistema canaletão apresentou comportamento linear na remoção acumulada de massa seca para todos os nutrientes (Figura 4), exceto para K que foi exponencial, enquanto a extração acumulada nos tubetes seguiu a mesma tendência.

Os conteúdos acumulados dos nutrientes removidos pelas minicepas (Figura 4) aos 273 DAS obedeceram a seguinte ordem decrescente para ambos os sistemas estudados: $\mathrm{N}>\mathrm{K}>\mathrm{Ca}>\mathrm{P}>\mathrm{Mg}>\mathrm{S}$.

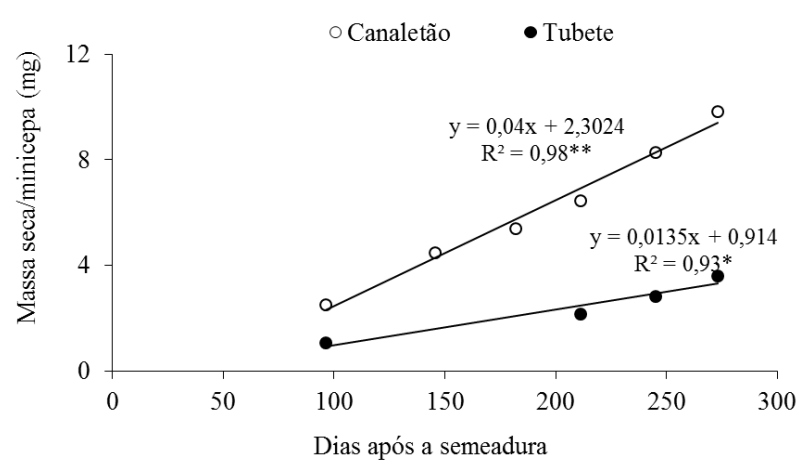

FIGURA 3: Massa seca removida no manejo de minicepas de Toona ciliata em dois sistemas de produção a partir da recepagem $(*$ significativo ao nível de $5 \%$ e $* *$ de $1 \%$ de probabilidade pelo teste " $\mathrm{t}$ " de Student).

FIGURE 3: Dry mass removed in the management of Toona ciliata ministumps in two production systems from the pruning (* values significant at $5 \%$ and $* * 1 \%$ probability by " $t$ " student test). 

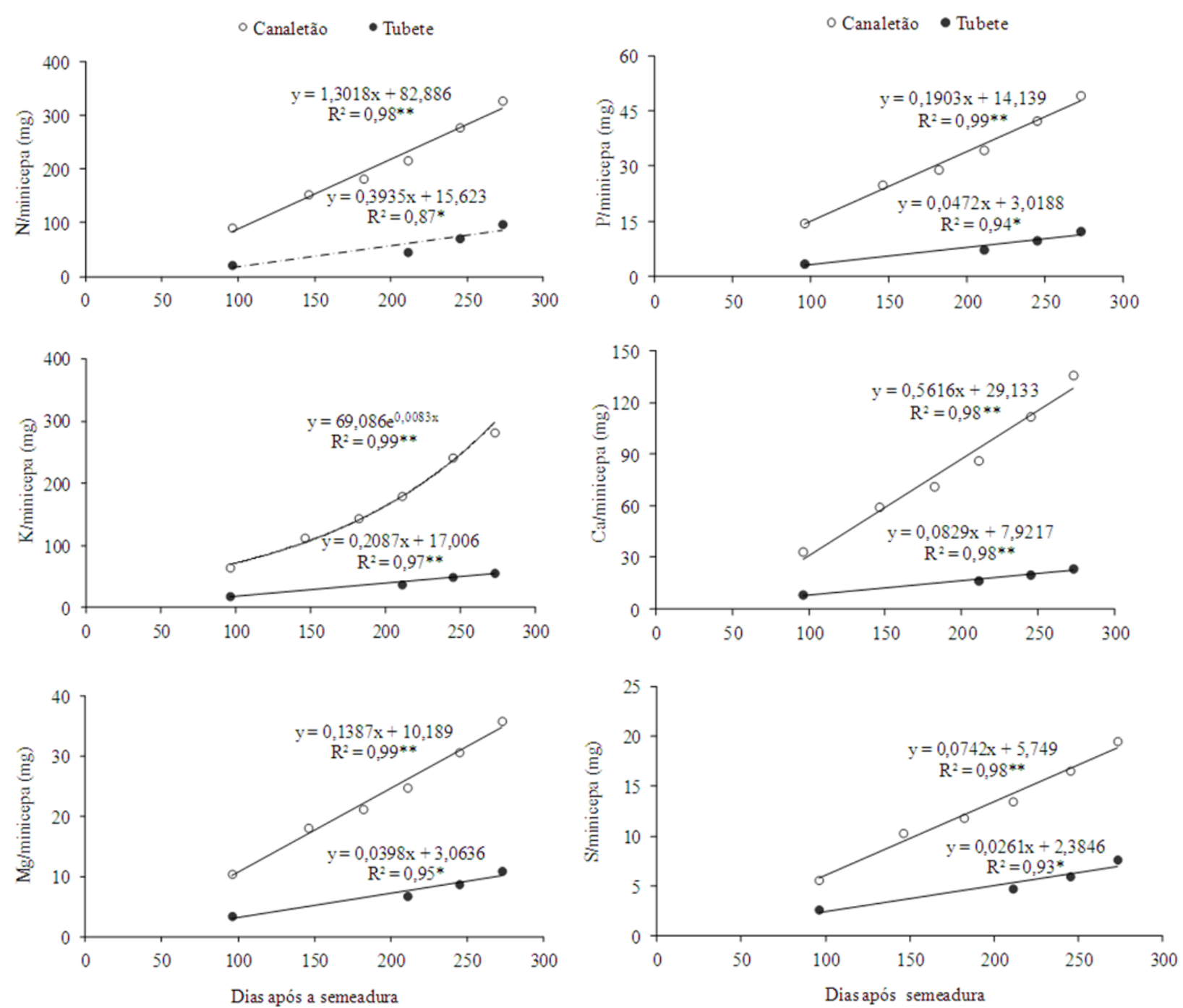

FIGURA 4: Conteúdo removido acumulado de N, P, K, Ca, Mg e S por minicepa de Toona ciliata no manejo de dois sistemas de produção a partir da recepagem (* significativo ao nível de $5 \%$ e ** de $1 \%$ de probabilidade pelo teste " $\mathrm{t}$ " de Student).

FIGURE 4: Accumulated content removal of N, P, K, Ca, Mg and $\mathrm{S}$ by ministumps of Toona ciliata in the management of two production systems from the pruning (* values significant at $5 \%$ and $* *$ $1 \%$ probability by " $t$ " student test).

No canaletão, as raízes têm a possibilidade de explorar maior volume de substrato adquirindo maior quantidade de nutrientes, o que reflete também sobre a produção de massa seca. Nos tubetes, por outro lado, mesmo a aplicação do sulfato de amônio não foi capaz de suprir a limitação imposta ao sistema radicular.

\section{CONCLUSÕES}

A produtividade das minicepas manejadas no sistema canaletão foi superior ao sistema tubete. Os nutrientes mais extraídos pelas minicepas no decorrer das coletas para ambos os sistemas de manejo foram o $\mathrm{N}$ e $\mathrm{K}$. O manejo das minicepas em canaletões resulta em extração de nutrientes, quando comparadas ao sistema em tubetes.

\section{REFERÊNCIAS BIBLIOGRÁFICAS}

AZEVEDO, E. B. et al. Substratos fertilizados com uréia revestida e o crescimento e estado nutricional da muda de citros. Acta Scientiarum. Agronomy, Maringa, v. 31, n. 1, p. 129-137, 2009.

CUNHA, A. C. M. C. M.; WENDLING, I.; JÚNIOR, L. S. Produtividade e sobrevivência de minicepas de Eucalyptus benthamii Maiden et Cambage em sistema de hidroponia e em tubete. 
Ciência Florestal. Santa Maria, v. 15, n. 3, p. 307310, 2005.

CUNHA, A. C. M. C. M.; WENDLING, I.; JÚNIOR, L.S. Miniestaquia em sistema de hidroponia e em tubetes de corticeira-do-mato. Ciência Florestal, Santa Maria, v. 18, n. 1, p. 85-92, 2008.

GARLET, T. M. B.; SANTOS, O. S. (2008) Solução nutritiva e composição mineral de três espécies de menta cultivadas no sistema Hidropônico. Ciência Rural, Santa Maria, v. 38, n. 5, p. 1233-1239, 2008. HIGASHI, E. N.; SILVEIRA, R. L. V. de A.; GONÇALVES, A. N. Propagação Vegetativa de Eucalyptus: Princípios Básicos e a sua Evolução no Brasil. Circular Técnica IPEF, n. 192, 2000. 10 p. HIGASHI, E. N.; SILVEIRA, R. L. V. de A.; GONÇALVES, A. N. Nutrição e Adubação em Minijardim clonal hidropônico de Eucalyptus. Circular Técnica IPEF, n. 194, 2002. 21 p.

LIAO, S. et al. Limonoids from the Leaves and Stems of Toona ciliata. Journal of Natural Products, Shanghai, v. 70, n. 8, p. 1268-1273, 2007. MALAVOLTA, E.; VITTI, G. C.; OLIVEIRA, S. A. Avaliação do estado nutricional das plantas, princípio e aplicações. 2. ed. Piracicaba: Potafo, 1997. 319 p.

MROGINSKI, E.; REY, H. Y.; MROGINSKI, L. A. (2003) In vitro plantlet regeneration from Australian Red Cedar (Toona ciliata, Meliaceae). New Forest, Netherlands, v. 25, p. 177-184, 2003.

MURAKAMI, C. H. G. Cedro Australiano: Valorização de Espécies Nobres Boletim Florestal, n. 7, 2008. p. 1-10.

PAIVA, Y. G. et al. Zoneamento agroecológico de pequena escala para Toona ciliata, Eucayptus grandis e Eucalyptus urophilla na Bacia Hidrográfica do Rio Itapemirim - ES, utilizando dados SRTM. In: SIMPÓSIOBRASILEIRO DESENSORIAMENTO REMOTO, 8., 2007, Florianópolis. Anais... INPE:
Florianópolis, 2007, p. 1785-1792.

PINHEIRO, A. L., LANI, L. L., COUTO, L. Cultura do Cedro Australiano para Produção de Madeira Serrada. Viçosa: UFV, 2003. 42 p.

ROSA, L. S. et al. Efeito da dose de nitrogênio e de formulações de substratos na miniestaquia de eucalyptus dunnii Maiden. Revista Árvore, Viçosa, v. 33, n. 6, p. 1025-1035, 2009.

SOUZA, J. C. A. V. et al. Propagação vegetativa de cedro-australiano (Toona ciliata M. Roemer) por miniestaquia. Revista Árvore, Viçosa, v. 33, n. 2, p. 205-213, 2009.

SOUZA JUNIOR, L.; QUOIRIN, M.; WENDLING, I. Miniestaquia de Grevillea robusta A. Cunn. a partir de propágulos juvenis. Ciência Florestal, Santa Maria, v. 18, n. 4, p. 455-460, 2008.

TITON, M. et al. Eficiência das minicepas e microcepas na produção de propágulos de clones de Eucalyptus grandis. Revista árvore, Viçosa, v. 27, n. 5, p. 619-625, 2003.

WENDLING, I.; DUTRA, L. F.; GROSSI, F. Produção e sobrevivência de miniestacas e minicepas de erva-mate cultivadas em sistema semihidropônico. Pesquisa Agropecuária Brasileira, Brasília, v. 42, n. 2, p. 289-292, 2007.

WENDLING, I.; FERRARI, M. P.; DUTRA, L. F. Produção de mudas de corticeira-do-mato por miniestaquia a partir de propágulos juvenis. Colombo/PR, 2005. 5 p. (Comunicado Técnico) WENDLING, I.; SOUZA JÚNIOR, L. Propagação vegetativa de erva-mate (Ilex paraguariensis Saint Hilaire) por miniestaquia de material juvenil. In: CONGRESSO SUL - AMERICANO DA ERVA-MATE, 2003, Chapecó. Anais..., Epagri: Chapecó, 2003. CD - rom.

XAVIER, A. et al. Propagação vegetativa de cedrorosa por miniestaquia. Revista Árvore, Viçosa, v. 27, n. 2, p. 139-143, 2003. 